\title{
KCNN4 Expression Is Elevated in Inflammatory Bowel Disease: This Might Be a Novel Marker and Therapeutic Option Targeting Potassium Channels
}

\author{
Christoph Süsss ${ }^{1,2}$, Lucile Broncy ${ }^{1}$, Kirstin Pollinger ${ }^{1}$, Claudia Kunst ${ }^{1}$, Karsten Gülow ${ }^{1}$, Martina Müller ${ }^{1}$, Gisela Wölfel ${ }^{1}$
}

1) Department of Internal

Medicine I, Gastroenterology,

Hepatology, Endocrinology,

Rheumatology and Infectious

Diseases, University Hospital

Regensburg, Regensburg;

2) Department of

Radiotherapy, University

Hospital Regensburg,

Regensburg, Germany

Address for correspondence:

Gisela Wölfel

Department of Internal

Medicine I, Gastroenterology,

Hepatology, Endocrinology,

Rheumatology and Infectious

diseases

University Hospital

Regensburg

Franz-Josef-Strauß-Allee 11

93053 Regensburg, Germany gisela.woelfel@klinik.uni-

regensburg.de

\begin{abstract}
Background \& Aims: The $\mathrm{K}^{+}$channel KCNN4 is involved in many inflammatory diseases. Previous work has shown that this channel is involved in epithelial ion transport and intestinal restitution. In inflammatory bowel diseases (IBD) a defective epithelial barrier can lead to typical symptoms like secretory diarrhea and the formation of intestinal ulcers. We compared surgical samples from patients with IBD, diverticulitis and controls without inflammation to determine the potential role of KCNN4 as a diagnostic marker and/or therapeutic target.

Methods: mRNA-levels of KCNN4 and a control $\mathrm{K}^{+}$channel were determined in intestinal epithelial cells (IEC) from patients with IBD, diverticulitis and controls. In addition, we performed a Western blot analysis of KCNN4 and a respective control $\mathrm{K}^{+}$channel in IEC from patients with IBD. Furthermore, we determined epithelial barrier integrity by measuring the flux of fluorescent-labeled dextran beads across a cell monolayer upon incubation with interferon- $\gamma$.

Results: KCNN4 mRNA and protein levels were elevated in IEC from patients with Crohn`s disease (CD) and ulcerative colitis (UC). Of note, KCNN4 was not elevated in non-IBD intestinal inflammatory conditions e.g. diverticulitis. Of clinical relevance, pharmacological KCNN4 channel openers stabilized epithelial barrier function in vitro. Thus, KCNN4 may have a protective role in IBD and constitute a therapeutic target.

Conclusions: Our data demonstrate elevated KCNN4 both at mRNA and protein level in IEC specifically from patients with IBD. Therefore, we conclude that KCNN4 could be used as a novel marker for IBD, especially for the establishment of initial diagnosis. Of therapeutic consequence, we show that pharmacological KCNN4 openers stabilize the epithelial barrier. Thus, KCNN4 might be a novel target to diagnose and treat IBD.
\end{abstract}

Key words: KCNN4 - potassium channels - marker of IBD - Crohn`s disease - ulcerative colitis - inflammatory bowel diseases.

Abbreviations: BCA: bicinchoninic acid; CD: Crohn`s disease; EGF: epidermal growth factor; EGFR: EGF receptor; IBD: inflammatory bowel disease; IEC: intestinal epithelial cells; IFN- $\gamma$ : interferon- $\gamma$; mRNA: messenger RNA; PCR: polymerase chain reaction; PI3K: phosphoinositide 3-kinase; PTPN2: protein-tyrosine phosphatase N2; Th: T helper; TNF: tumor necrosis factor; UC: ulcerative colitis.

\section{INTRODUCTION}

Despite many years of intensive research, the exact etiologies of the two entities of chronic inflammatory bowel diseases (IBD), Crohn's disease (CD) and ulcerative colitis (UC), remain unclear. Current hypotheses suggest that genetically predisposed individuals develop an exaggerated response to bacterial components in the gastrointestinal tract, translating to an excessive immune reaction towards the commensal flora. The etiology is multifactorial, and many gene loci have been found to serve as susceptibility genes for different subgroups of patients with IBD $[1,2]$. Environmental influences are also considered as factors of IBD onset. Recent data highlight the role of intestinal flora composition in both initiation and propagation of chronic inflammation $[3,4]$. In CD a predominant T helper 1 (Th1)-immune response has been described, whereas in UC a predominant T helper 2 (Th2)-immune response has been identified [5]. Interferon- $\gamma($ IFN- $\gamma$ ) plays a pivotal role in different models of murine colitis, especially in the commonly used dextran sulfate sodium-induced murine colitis [6] as well as in CD, where IFN- $\gamma$ levels are elevated in 
serum and mucosa $[7,8]$. IFN- $\gamma$ and other proinflammatory cytokines such as the tumor necrosis factor (TNF) can disrupt tight junctions and cause epithelial damage leading to a leaky mucosal barrier [9]. Persistent levels of inflammatory cytokines mediate impairment of tight junction and apoptosis of intestinal epithelial cells (IEC) [10], which increase epithelial permeability [11]. The epithelial barrier function in the intestinal tract is not only essential for regulated ion transport processes of the epithelium, but also for wound healing. During inflammation, superficial wounds occur constantly and are quickly restored by intestinal epithelial restitution [12]. This process is supported by different growth factors, including epidermal growth factor (EGF) that promotes epithelial migration of intestinal cells [13].

Our research group [14] and others [10] investigated the role of the intestinal epithelial monolayer in chronic intestinal inflammation with overproduction of IFN $-\gamma$. We described a defective EGF receptor (EGFR) activation in IEC with a shift towards the phosphoinositide 3-kinase (PI3K) pathway caused by IFN- $\gamma[14]$.

Alterations in downstream signaling pathways originating at EGFR play a pivotal role for ion transport responses as well as for intestinal restitution. Preincubation of IEC with IFN- $\gamma$ exerts a direct influence on anion secretion by abrogating the inhibitory effect of EGF on carbachol-induced $\mathrm{K}^{+}$currents [15]. These data demonstrate that $\mathrm{Ca}^{2+}$-dependent $\mathrm{K}^{+}$currents are altered in intestinal inflammation when elevated IFN- $\gamma$ levels are present. Basolateral $\mathrm{K}^{+}$currents are the driving force for apical sodium secretion $[16,17]$. KCNN4 is a predominantly basolateral located $\mathrm{Ca}^{2+}$-activated $\mathrm{K}^{+}$channel that is not only involved in ion transport responses in inflammation, but also in epithelial restitution $[12,13,18,19]$. The epithelial barrier function maintains electrical gradients necessary for controlled regulation of absorption vs. secretion of electrolytes and water in the healthy intestine. A disturbed balance between absorption and secretion of the intestinal epithelium can lead to secretory diarrhea, one of the major symptoms of both CD and UC. In earlier studies, we observed a disruption of the actin cytoskeleton after prolonged IFN- $\gamma$ treatment of the epithelial cell monolayer $[14,20]$. This may constitute an alternative mode of action of IFN- $\gamma$ in addition to the disruption of tight junctions. Of note and of clinical relevance, both mechanisms lead to a leaky epithelial barrier.

A defective epithelial barrier with enhanced permeability of the intestinal epithelium seems to be involved in the development of diarrheal symptoms primarily in $\mathrm{CD}$. Further data indicate that a reduced $\mathrm{K}^{+}$conductance in UC leads to reduced sodium absorption that could account for diarrheal symptoms [21]. In addition, defects in epithelial integrity together with an excessive immune response to the commensal flora may initiate chronic intestinal inflammation and trigger the onset of UC and CD [22]. Proinflammatory cytokines, predominantly IFN- $\gamma$, are elevated in the mucosa of CD patients. Several genes involved in the development of IBD seem to influence permeability of the epithelial barrier in conjunction with IFN- $\gamma$ secretion [23]. In an earlier study we investigated the role of the $\mathrm{CD}$ candidate gene proteintyrosine phosphatase N2 (PTPN2) in maintaining the epithelial barrier function after preincubation with IFN- $\gamma$. PTPN2 plays a protective role by reducing IFN- $\gamma$-induced signaling and consequent induction of barrier defects [24]. Furthermore, earlier studies from our group revealed a prominent role of $\mathrm{KCNN} 4$ in $\mathrm{Ca}^{2+}$-dependent ion transport and intestinal restitution [14]. In a T cell-mediated mouse model of colitis, inhibition of KCNN4 ameliorated the disease [24]. Increased epithelial permeability has been described as an important pathophysiological mechanism for IBD [21]. Defective tight junctional complexes mediate dysfunctions of the epithelial barrier. It has been shown that $\mathrm{K}^{+}$channels have an impact on tight junctions in epithelial cells $[25,26]$. However, the role of KCNN4 in this network has not been investigated. It is known that $\mathrm{K}^{+}$channels are involved in intestinal restitution.

We have previously demonstrated that the inhibition of the $\mathrm{Ca}^{2+}$-activated $\mathrm{K}^{+}$channel KCNN4 differentially regulates IEC migration in inflamed vs. non-inflamed conditions and that migration is mediated by the PI3K signaling pathway [14]. The apical and basolateral $\mathrm{K}^{+}$channel KCNN4 has been shown to be involved in different inflammatory diseases e.g. IBD and chronic inflammatory airway disease $[26,27]$. KCNN4 is able to enhance the proliferative response of mesenteric lymph nodes in an experimental mouse model of colitis [28]. The basolateral cAMP-dependent $\mathrm{K}^{+}$channel KCNQ1 is abundant in the intestine and was proven to be essential for intestinal chloride transport [29]. Accordingly, $\mathrm{K}^{+}$channel KCNQ1 is a suitable control to further characterize the $\mathrm{Ca}^{2+}$-activated $\mathrm{K}^{+}$channel KCNN4 in IEC. $\mathrm{K}^{+}$channels interact with growth factor-dependent signaling cascades during intestinal epithelial restitution. Elevated $\mathrm{K}^{+}$channel expression has been shown in different cell types in the mucosa of patients with ileal CD [30]. However, the clinical relevance of this finding has not been analyzed so far. Thus, we aimed to analyze the expression of KCNN4 at mRNA and protein level in IEC isolated from patients with IBD compared to controls in order to investigate whether this channel could serve as a potential clinical marker for IBD. Since we had the opportunity to analyze intestinal tissue specimens from bowel resections, we were able to isolate and analyze primary IEC from a cohort of 60 patients with CD and UC regarding KCNN4 expression. In addition, we investigated whether KCNN4 constitutes a novel potential therapeutic target by monitoring the impact of channel inhibitors and openers on epithelial barrier integrity.

\section{METHODS}

\section{Cell culture}

The newborn rat non-transformed IEC line IEC-18 (ATCC CRL-1589; passages 25-55) was purchased from ATCC (Manassas, VA, USA). The IEC-18 cell line is derived from ileal crypt cells [31]. IEC-18 cells were cultured in DMEM supplemented with $4.5 \mathrm{~g} / \mathrm{l}$ glucose, L-glutamine (PAA, Pasching, Austria), 5\% FCS (Sigma Aldrich, Steinheim, Germany), $100 \mathrm{U} / \mathrm{ml}$ penicillin, $100 \mu \mathrm{g} / \mathrm{ml}$ streptomycin (PAA, Pasching, Austria) and $0.7 \mathrm{mM}$ bovine insulin (Sigma-Aldrich, Steinheim, Germany) in a humidified atmosphere at $37^{\circ} \mathrm{C}$ with $10 \%$ CO2 [32, 33]. Sub-cultivation of IEC-18 was carried out twice per week in a ratio of 1:6. Fully supplemented cell culture medium was routinely filtered through steriflip filters (Millipore, Billerica, MA, USA). 


\section{Isolation of primary intestinal epithelial cells}

We obtained 60 surgical specimens from patients with CD, UC, sigmoid diverticulitis and colorectal carcinoma neoplasia (> $10 \mathrm{~cm}$ distance from the tumor) after written informed consent. The Ethics Commission at the University of Regensburg had accepted a specific request (record number 00/14) and the procedure was performed according to the Declaration of Helsinki. Intestinal epithelial cells were isolated as previously described $[34,35]$.

\section{RNA isolation and real-time-PCR Analysis}

We used the RNeasy Mini Kit (Qiagen`, Hilden, Germany) to isolate RNA according to the manufacturer's instructions. cDNA was synthesized using affinity script reagents (Agilent Technologies GmbH, Böblingen, Germany) as given in the manufacturer's protocol. Real-time-PCR was executed by using 2x Brilliant ${ }^{\circledast}$ II Mastermix qPCRHigh ROX (Agilent Technologies GmbH, Böblingen, Germany) and Human GAPDH-Mix (20x) (Life Technologies, Carlsbad, USA) with Light Cycler ${ }^{\circledR} 80$ (Roche Diagnostics Deutschland GmbH, Mannheim, Germany) according to the manufacturer's specifications.

Primer sequences were as follows: human KCNN4 forward 5 '-CCC TCA TCA AAA ACA CTC TCA CTA TG-3', reverse 5 - TCC AGT CGC CTG CAC TTG-3`, probe 5 ` - FAM-TGC TAT GGA CGA CCT CCA GCT CTC AGT T-TAMRA-3', human KCNQ1 forward 5 ' - CGC ATG GAG GTG CTA TGC T-3`, reverse 5` - GGC CTT CCG GAT GTA GAT CTT-3`, probe $5^{`}$ - FAM-AGA ACC CCG ACT CCT CCA CCTTAMRA-3' (all from Eurofins MWG Operon, Ebersberg, Germany).

Human GAPDH mix was used as endogenous control (Applied Biosystems, Carlsbad, CA, USA). PCR conditions were two minutes of incubation at $50^{\circ} \mathrm{C}$, followed by ten minutes at $95^{\circ} \mathrm{C}$ and 40 cycles for 15 seconds each at $95^{\circ} \mathrm{C}$ for denaturation and 60 seconds at $60^{\circ} \mathrm{C}$ for annealing and extension each. Analysis of the results was performed by the delta-delta cycle threshold (ddCT) method [36].

\section{Western Blot analysis}

Primary IEC were isolated as described above. After removal of the crypts, cell pellets were resuspended in 300$750 \mu l$ RIPA-buffer (Sigma Aldrich Chemie GmbH, Steinheim, Germany). The suspension was centrifuged at $13000 \mathrm{rpm}$, the clear supernatant was collected and stored at $-20^{\circ} \mathrm{C}$. BCA (bicinchoninic acid) assay was used for measuring protein concentrations.

Volumes containing $7.5 \mu \mathrm{g}$ protein each were loaded on a $4-12 \%$ polyacrylamide gel to separate proteins by electrophoresis. Proteins were then transferred onto nitrocellulose membranes. NuPage instruments and reagents were used according to the manufacturer's instructions (all from Invitrogen, Carlsbad, CA). Membranes were blocked with 5\% BSA (Biomol, Hamburg, Germany) in a Trisbuffered saline with $0.1 \%$ Tween 20 (TBST) (Sigma-Aldrich, St Louis, MO, USA) overnight, followed by incubation with the respective primary antibody diluted in blocking buffer: rabbit anti-KCNN4 polyclonal antibody (Bioss, Woburn, MA,
USA) 1:500 or rabbit anti-KCNQ1 polyclonal antibody (Bioss, Woburn, MA, USA) 1:200 and for loading control anti- $\beta$-actin (Sigma Aldrich Chemie GmbH, Steinheim, Germany). As a secondary antibody we used anti-rabbit-IgG-HRP (Santa Cruz Biotechnology, Heidelberg, Germany) (1:3000 in 10 $\mathrm{ml} 2.5 \%$ milk/2.5\% BSA). Membranes were exposed to $5 \mathrm{ml}$ of enhanced chemiluminescence (ECL)-solution $(2.5 \mathrm{mM}$ luminol, 0.1 M Tris- $\mathrm{HCl}$ and $0.4 \mathrm{mM}$ p-coumarin acid - all from Sigma-Aldrich Chemie GmbH, Steinheim, Germany in aqua dest.) mixed with $1.53 \mu \mathrm{l}$ of $30 \%$ hydrogen peroxide (Merck, Darmstadt, Germany) for five minutes. Detection of immunoreactive proteins was performed by using a Biorad Imager and Image Lab ${ }^{\text {tm }}$ Software. For multiple use, membranes were stripped using ReBlot-plus strong solution (Chemicon, Temecula, CA, USA).

\section{Permeability assay}

Transepithelial permeability was assessed by measuring fluorescein isothiocyanate-dextran (FITC-dextran, 40,000 mmol, Sigma Aldrich Chemie GmbH, Steinheim, Germany) flux across IEC-18 cell monolayers. 200,000 cells per well were seeded into Millicell Cell Culture Inserts (Merck Millipore $\mathrm{GmbH}$, Billerica, MA, USA), which were placed in the wells of a 24-well plate (Costar Corning Incorporated, Corning, NY, USA). IEC monolayers were preincubated for 24 and 48 hours with IFN- $\gamma$. As pharmacological $\mathrm{K}^{+}$ channel modulators we used the KCNN4 opener SKA-31 at a concentration of $5 \mu \mathrm{M}$ [37] as well as the KCNN4 opener 1 -EBIO at a concentration of $600 \mu \mathrm{M}$ [38]. Moreover, we used the following KCNN4 inhibitors: TRAM-34 $(25 \mu \mathrm{M})$ $[39,40]$ and clotrimazole $(10 \mu \mathrm{M})$ [39]. Clotrimazole is an FDA-approved KCNN4 channel blocker and cytochrome P450 inhibitor. TRAM-34 is an analogue of clotrimazole which has no impact on cytochrome P450 [41]. In addition, the KCNMA1 blocker iberiotoxin $(100 \mathrm{nM})$ was used to examine possible unspecific effects modulated by other $\mathrm{K}^{+}$ channels $[42,43]$.

After the respective treatment, IEC-18 cells were washed three times with Ringer's solution. A dilution $(1.25 \mathrm{mg} / \mathrm{ml})$ of FITC-dextran was mixed and $100 \mu \mathrm{l}$ of this solution were added to the apical side of the monolayer in each insert [44, 45]. After incubation periods of 30 minutes and 1 hour, 100 $\mu \mathrm{l}$ of the basolateral solution was removed and fluorescence was measured using a Tecan Spectra Fluor fluorescence and absorbance reader (Tecan Group AG, Männedorf, Switzerland). Molecules were excited at $485 \mathrm{~nm}$ and the emission was measured at $535 \mathrm{~nm}$.

\section{Statistical analysis}

For statistical analysis and creating diagrams IBM SPSS Statistics 20 was used. The Kolmogorov-Smirnov-test was performed for normal distribution and the Mann-WhitneyU-test was applied for calculation of p-values. Statistical p-values of 0.05 or less were considered to be significant. Outliers were eliminated after performing the outlier test by Grubbs.

Data analyzing protein levels are shown as $\mathrm{x}$-fold expression relative to the mean of the control group. 


\section{RESULTS}

mRNA levels of potassium channel $K C N N 4$ in intestinal epithelial cells isolated from surgical specimens of patients with IBD

To analyze the clinical significance of the $\mathrm{Ca}^{2+}$-activated $\mathrm{K}^{+}$ channel KCNN4 in human IBD, we isolated IEC from surgical specimens of patients with IBD (and other disease entities as controls) and quantified the expression of KCNN4. In total, we analyzed mRNA data from IEC of 60 patients. Subgroups were composed of specimens from patients with CD $(n=28)$, UC $(n=14)$, colorectal carcinoma $(n=10)$ and diverticulitis $(n=8)$. Table I provides an overview of the patients enrolled in our study. In macroscopically inflamed tissue from UC $(n=14)$ and $\mathrm{CD}(\mathrm{n}=28)$ patients, KCNN4 expression in IEC was increased compared to respective controls from colorectal carcinoma without macroscopically detected inflammation $(n=10)$. KCNN4 transcription was significantly upregulated in UC (mean 658.2 $\pm 158.7 \% ; \mathrm{n}=14$ ) compared to colorectal carcinoma $(0 \pm 37,8 \% ; \mathrm{n}=10)$ as well as in $\mathrm{CD}(415.6 \pm 124.1 \%)$ compared to colorectal carcinoma (Fig. 1A). In diverticulitis (662.7 $\pm 511.8 \%)$ no significant differences were observed, neither in comparison to colorectal carcinoma nor in comparison to CD nor to UC (Fig. 1A). Expression of KCNQ1, a voltage-gated $\mathrm{K}^{+}$channel, was used as control. Levels of KCNQ1 mRNA were comparable in all groups tested (Fig. 1B). There was no significant change observed in UC $(-24.65 \pm 136.6 \%)$ compared to colorectal carcinoma $(p=0.212)$, in CD $(21.5 \pm 23.9 \%)$ compared to colorectal carcinoma $(\mathrm{p}=0.708)$ and in diverticulitis $(256.4 \pm 176.5 \%)$ compared to colorectal carcinoma $(\mathrm{p}=0.762)$ (Fig. 1B). Therefore, a general effect on $\mathrm{K}^{+}$channel expression mediated by inflammatory responses is unlikely.

\section{KCNN4 protein level in patients with IBD}

We investigated the KCNN4 protein content in IEC from patients with $\operatorname{IBD}(n=6)$, diverticulitis $(n=4)$ and controls with colorectal carcinoma $(n=3)$. To distinguish between inflamed and non-inflamed conditions we compared all patients with intestinal inflammation (UC, CD and diverticulitis) to controls without inflammation (colorectal carcinoma). Of clinical relevance, we could show a significant upregulation of KCNN4 protein content in IEC from patients with intestinal inflammation $(63.8 \pm 22.0 \% ; \mathrm{n}=10)$ compared to controls $(0 \pm 5.5 \% ; n=3)$ (Figs. $2 \mathrm{~A}$ und $2 \mathrm{~B})$. The content of control protein KCNQ1 was not altered in all tested groups $(2.8 \pm 5.0 \% ; n=10)$ (Figs. 3A and 3B).
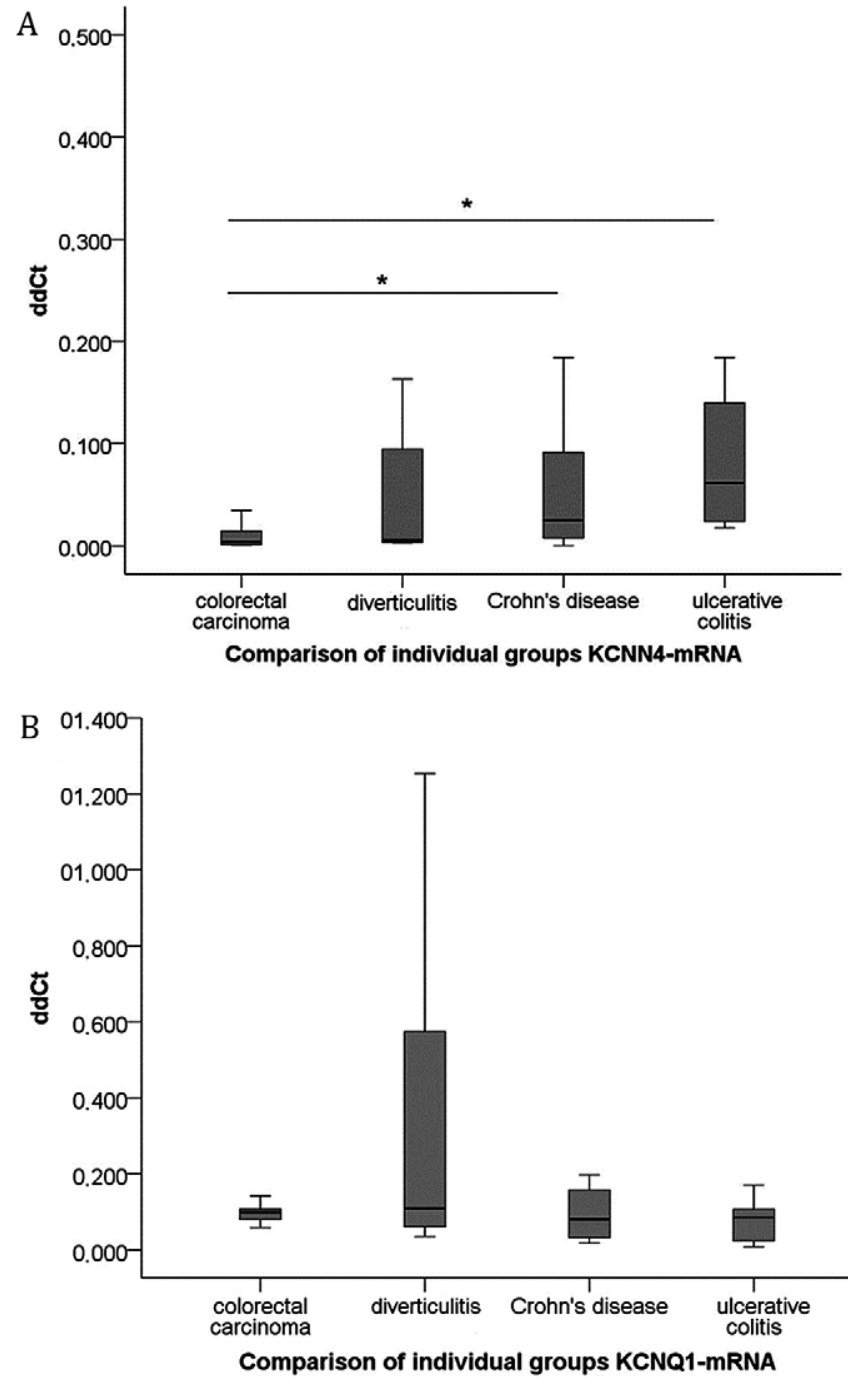

Fig. 1. KCNN4 mRNA levels are elevated in IBD compared to controls. A) KCNN4 mRNA expression, shown as absolute ddCt values (all performed in triplicate), was analyzed in IEC from patients with $\mathrm{CD}$ $(\mathrm{n}=28)$, UC $(\mathrm{n}=14)$, diverticulitis $(\mathrm{n}=8)$ and controls (colorectal carcinoma) $(\mathrm{n}=10)$. B) KCNQ1 mRNA expression, shown as absolute ddCT values (all performed in triplicate) in IEC from the same patient groups. Data are expressed as -fold control. Asterisks denote statistically significant differences from control $\left({ }^{*}, \mathrm{p} \leq 0.05\right)$.

Targeting of KCNN4 by potassium channel openers on epithelial barrier function

Monolayers of IEC-18 cells were preincubated for 24 and 48 hours with IFN- $\gamma$. To analyze permeability, we measured

Table I. Patients characteristics

\begin{tabular}{|c|c|c|c|c|c|}
\hline \multirow[t]{2}{*}{ Patients characteristics } & & \multicolumn{4}{|c|}{ Diagnosis } \\
\hline & & $\begin{array}{l}\text { Ulcerative } \\
\text { colitis }\end{array}$ & $\begin{array}{l}\text { Crohn's } \\
\text { disease }\end{array}$ & $\begin{array}{c}\text { Sigmoid } \\
\text { diverticulitis }\end{array}$ & $\begin{array}{l}\text { Colorectal } \\
\text { carcinoma }\end{array}$ \\
\hline Samples of patients, N (\%) & $60(100.0 \%)$ & $14(23.3 \%)$ & $28(46.7 \%)$ & $8(13.3 . \%)$ & $10(16.7 \%)$ \\
\hline \multicolumn{6}{|l|}{ Gender N (\%) } \\
\hline female & $31(51.7 \%)$ & $5(8.3 \%)$ & $18(30.0 \%)$ & $5(8.3 \%)$ & $3(5.0 \%)$ \\
\hline male & $29(48.3 \%)$ & $9(15.0 \%)$ & $10(16.7 \%)$ & $3(5.0 \%)$ & $7(11.7 \%)$ \\
\hline \multicolumn{6}{|l|}{ Age at surgical intervention } \\
\hline years mean & 47.9 & 40.5 & 39.7 & 68.9 & 64.2 \\
\hline
\end{tabular}


FITC-dextran flux across IEC-18 monolayers. After 24 hours preincubation with IFN- $\gamma$ no significant difference in barrier permeability was detected $(0.6 \pm 5.0 \%$; $\mathrm{p}=0.487$; Supplementary file Fig. 1). However, a significantly elevated permeability of the barrier was detected 48 hours after stimulation with IFN- $\gamma$ (24.1 $\pm 5.6 \%$; $=0.031$ ) (Fig. 4), showing that a prolonged incubation with IFN- $\gamma$ is needed to reduce epithelial monolayer integrity.
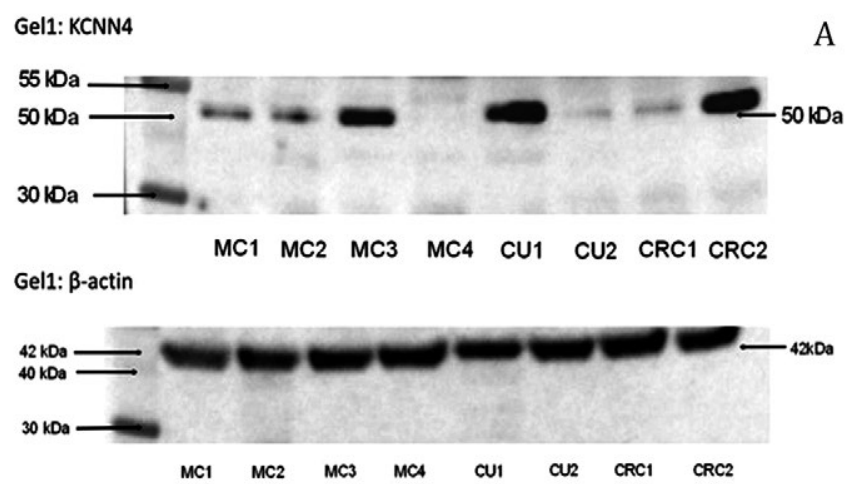

Gel2: KCNN4
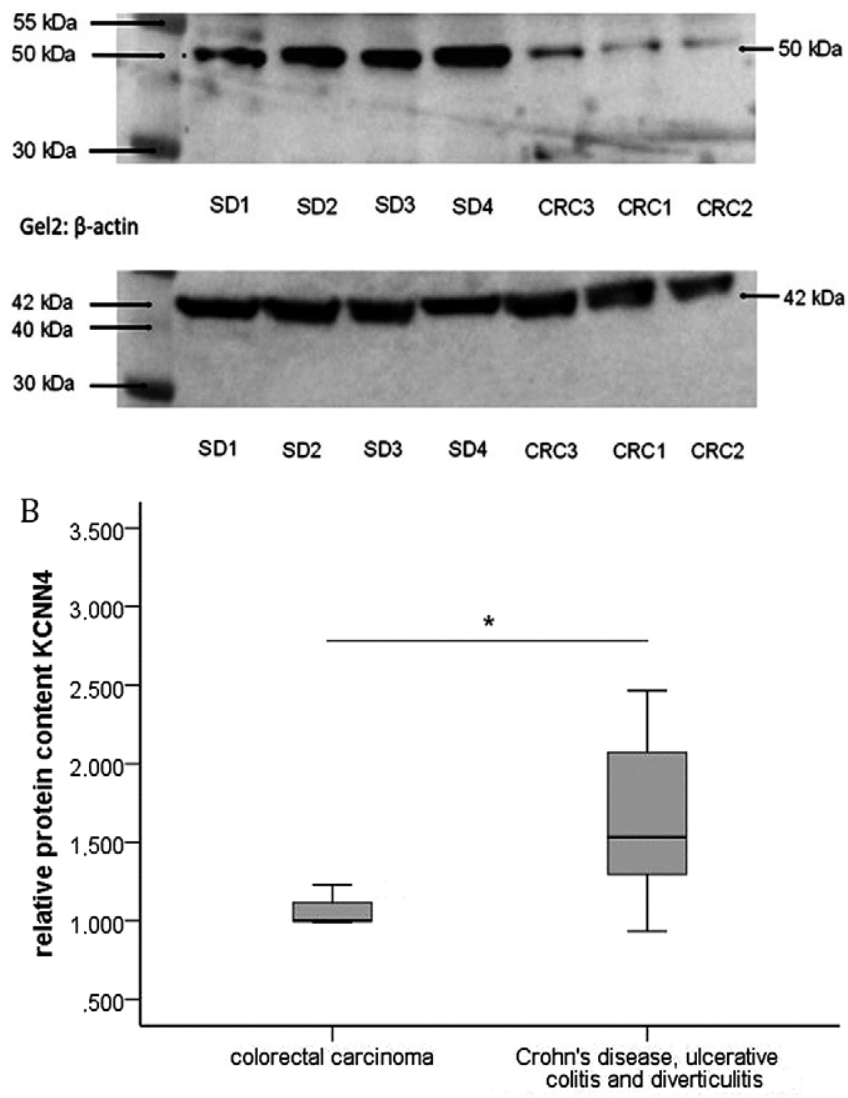

Comparison of Crohn's disease, ulcerative colitis and diverticulitis to colorectal carcinoma

Fig. 2. KCNN4 protein levels are elevated in intestinal inflammation compared to uninflamed controls A) representative Western blot of whole cell lysates from primary IEC from patients with CD $(n=4)$, UC $(n=2)$, diverticulitis $(n=4)$ and colorectal carcinoma $(n=3)$, sequentially probed for KCNN4 or $B$-actin as loading control. B) Densitometric analysis reveals significantly elevated KCNN4 levels in samples from patients with intestinal inflammation (CD, UC and diverticulitis together) compared to colorectal carcinoma as uninflamed controls. Data are expressed as -fold control. Asterisks denote statistically significant differences from controls $\left({ }^{*}, \mathrm{p} \leq 0.05\right)$.
Gel1: KCNQ1

A

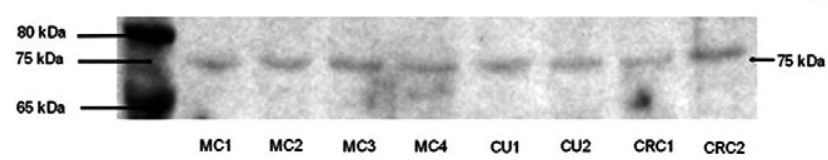

Gel1: $\beta$-actin

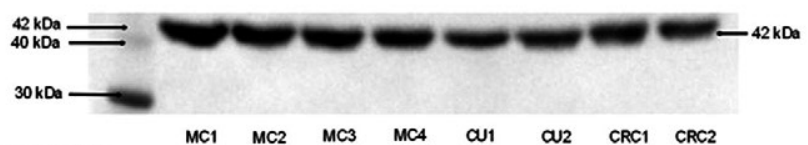

Gel2: KCNQ1

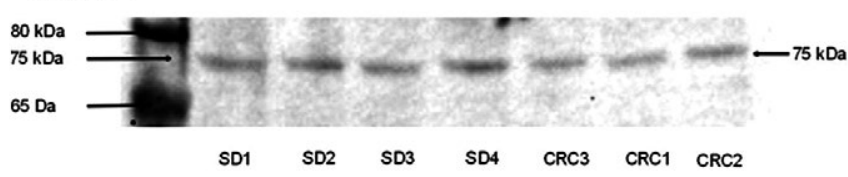

Gel2: $\beta$-actin
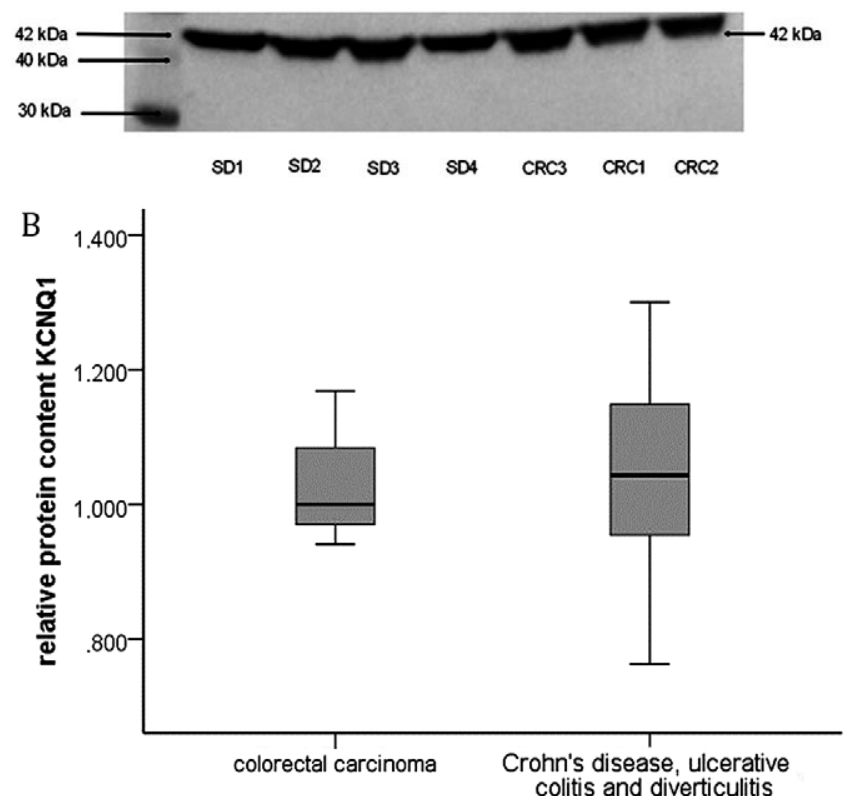

Comparison of Crohn's disease, ulcerative colitis and diverticulitis to colorectal carcinoma

Fig. 3. KCNQ1 protein levels as controls are similar in all groups A) representative Western blot of whole cell lysates from primary IEC from patients with CD $(\mathrm{n}=4)$, UC $(\mathrm{n}=2)$, diverticulitis $(\mathrm{n}=4)$ and colorectal carcinoma $(n=3)$, sequentially probed for KCNQ1 or $B$-actin as loading control. B) Densitometric analysis reveals similar KCNQ1 expression in samples from patients with intestinal inflammation (CD, UC and diverticulitis together) and in samples from patients with colorectal carcinoma as uninflamed controls. Data are expressed as -fold control. Asterisks denote statistically significant differences from controls $(\mathrm{p} \leq 0.05)$.

We titrated TRAM-34 to define the optimal concentration for our experiments. For concentrations of $0.5 \mu \mathrm{M} 1 \mu \mathrm{M}$, $10 \mu \mathrm{M}$ and $25 \mu \mathrm{M}$ no differences on membrane integrity were observed (Supplementary file Fig. 2). The KCNMA1 inhibitor iberiotoxin was used as a control [45]. In addition, two KCNN4 channel openers, namely 1-EBIO and SKA-31 were applied to evaluate $\mathrm{KCNN} 4$ as a potential therapeutic target.

The two KCNN4 inhibitors TRAM-34 (0.5 $\pm 26.1 \%$; $\mathrm{p}=0.51)$ and clotrimazole $(11.7 \pm 32.5 \%$; $=0.51)$ as well as the KCNMA1 inhibitor iberiotoxin $(16.1 \pm 47.4 \%$; $=0.51)$ did not influence the permeability compared to the respective control (Fig. 4). 


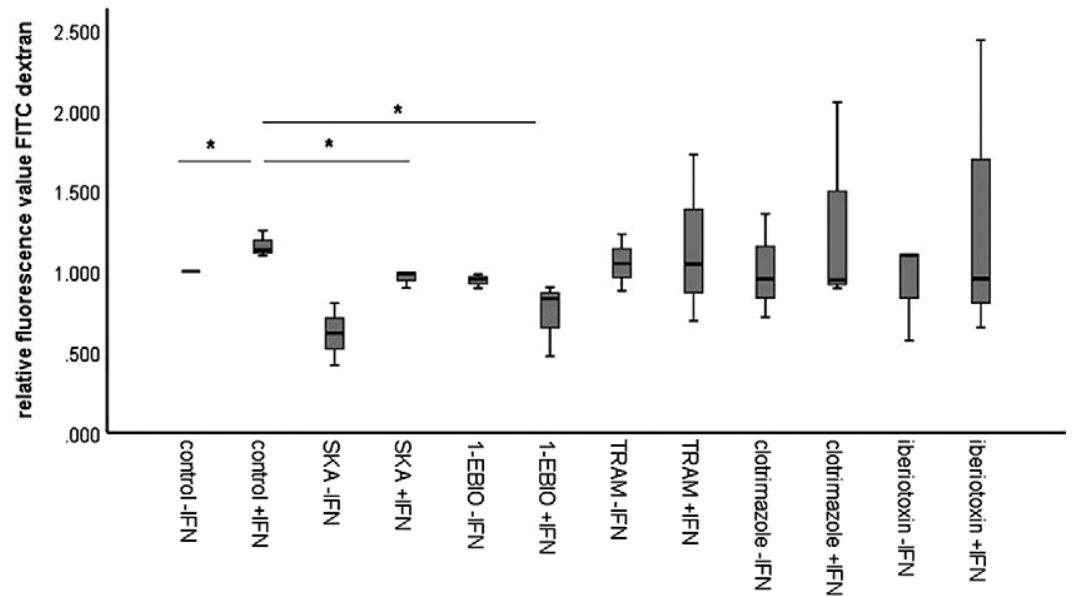

Fig. 4. FITC dextran flux in IEC18 cells after 48 hours of IFN- $\gamma$ incubation is significantly elevated. The KCNN4 channel opener SKA-31 and the K+ channel opener 1-EBIO lead to a significantly reduced FITC dextran flux after 48 hours of IFN- $\gamma$ incubation. Two KCNN4 inhibitors (TRAM-34 and clotrimazole) and a KCNMA1 inhibitor (iberiotoxin) have no significant influence on the FITC dextran flux after IFN- $\gamma$ incubation (all performed in triplicate). Data are expressed as -fold control. Asterisks denote statistically significant differences from control $\left({ }^{\star}, \mathrm{p} \leq 0.05\right)$.

Of clinical relevance, the KCNN4 opener SKA-31 significantly reduced permeability of the cell monolayer $(-17.3 \pm 2.7 \% ; \mathrm{p}=0.05)$. An even stronger effect was observed after addition of 1 -EBIO (-36.7 $\pm 11.5 \%$; $=0.05)$ (Fig. 4). Thus, KCNN4 channel openers significantly improve gut epithelial monolayer integrity.

\section{DISCUSSION}

KCNN4 is the $\mathrm{K}^{+}$channel which is expressed most prominently in the basolateral cell membrane of human IEC [46]. Recent data show that $\mathrm{K}^{+}$channels are involved in the pathogenesis of chronic inflammation such as IBD or chronic airway disease [47]. The role of KCNN4 in IBD is controversially discussed since this $\mathrm{K}^{+}$channel is expressed in different cell types such as Paneth cells, immune cells and IEC [48-50]. The impact of KCNN4 expression or activity was extensively analyzed in immune cells. T cell activation is altered by the expression level and activity of KCNN4 in T lymphocytes in pediatric patients with IBD [51]. The expression level of KCNN4 can be restored by anti-TNF therapy in these patients [52]. KCNN4 expression in T cells can be correlated with disease activity in UC [48]. Similarly, elevated T cell KCNN4 expression in UC correlates with inflammatory cytokines levels and disease activity [49]. In CD a gene variant of KCNN4 has been described that is associated with ileal disease in the New Zealand and Australian population [30]. In a T-cell-induced mouse model of colitis it has been shown that a reduced KCNN4 activity in T-lymphocytes leads to a minor degree of inflammation [53].

We have demonstrated previously that inhibition of the $\mathrm{Ca}^{2+}$-activated $\mathrm{K}^{+}$channel KCNN4 differentially regulates IEC migration in inflamed vs. non-inflamed conditions and that this is mediated by the PI3K signaling pathway [14]. Moreover, basolateral $\mathrm{K}^{+}$channels function as the driving force for apical chloride secretion in IEC. It is therefore of special therapeutic interest if the expression of this channel is modulated in IEC from patients with IBD.

In this study, we analyzed the expression of the $\mathrm{Ca}^{2+}$ activated $\mathrm{K}^{+}$channel KCNN4 in IEC isolated from surgical specimens of patients with IBD and controls. We evaluated the expression of KCNN4 at mRNA and protein level in IEC from patients with $\mathrm{CD}, \mathrm{UC}$, diverticulitis and non-inflamed controls. In our experiments KCNN4 mRNA was significantly increased in IEC from patients with chronic IBD, but not in patients with diverticulitis or colorectal carcinoma. This suggests an involvement of KCNN4 in development and course of chronic IBD. Moreover, not only KCNN4 mRNA level, but also KCNN4 protein content was also augmented in IEC from patients with intestinal inflammation, whereas KCNQ1 protein content was not altered in all groups. This result emphasizes the importance of the $\mathrm{Ca}^{2+}$-activated $\mathrm{K}^{+}$channel KCNN4 for IBD pathogenesis. As expression of KCNN4 is specifically elevated in patients with IBD, this could make KCNN4 an important and novel therapeutic target and/or diagnostic marker for IBD.

In IBD, epithelial barrier function is impaired and inflammatory cytokines such as IFN $-\gamma$ are thought to mediate these alterations in the barrier function. IFN- $\gamma$ is increased in $\mathrm{CD}$ in the intestinal epithelium [8]. We described earlier that IFN- $\gamma$ causes a defective EGFR activation in IEC during inflammation. Alterations in downstream signaling pathways originating at EGFR play a pivotal role for ion transport responses as well as for intestinal restitution. We demonstrated that inhibition of KCNN4 leads to enhanced migration of IEC in non-inflamed tissues. However, in inflamed tissues, this effect was almost prevented [14]. Downregulation of tight junction proteins plays a central role in the destabilization of the barrier. It has been shown that $\mathrm{K}^{+}$channels have an impact on tight junctions of epithelial cells $[25,26]$. However, the exact role of KCNN4 in regulating epithelial barrier integrity needs to be elucidated. Since we had shown elevated KCNN4 levels in primary IEC of patients with IBD, we investigated whether 
this $\mathrm{K}^{+}$channel may display protective properties for epithelial barrier in IBD.

In this study we performed preincubation with IFN- $\gamma$ in order to mimic inflammation in IEC-18-cells. This model was developed based on published data of other groups to investigate barrier function of cell monolayers following IFN- $\gamma$ treatment $[44,45,54]$. As expected, we observed an increased permeability of the cell monolayer upon IFN- $\gamma$ treatment. Remarkably and especially of clinical relevance, after addition of channel openers (e.g. 1-EBIO and SKA-31) epithelial barrier function was restored. Our data underline the important role of KCNN4 as a therapeutic target to stabilize epithelial barrier integrity in intestinal inflammation. In contrast, pharmacological inhibition of this $\mathrm{K}^{+}$channel revealed no change of the permeability of the epithelial monolayer. A different $\mathrm{K}^{+}$channel, the TWIK related $\mathrm{K}^{+}$channel 1 (Trek 1) affects the epithelial barrier, but its expression is reduced by allergic responses [55].

\section{CONCLUSION}

Our data show that KCNN4 mRNA expression is specifically increased in IBD but not in other intestinal inflammations e.g. diverticulitis. In clinical practice there is a diagnostic gap in some instances at the time of first diagnosis of IBD. In some cases, histologic results after the first endoscopy are unspecific. The lack of a reliable distinction between IBD and unspecific acute colitis (e.g. infectious colitis) often results in a delay of a specific therapy. In this study we show that KCNN4 mRNA serves as a novel diagnostic marker for IBD which can be analyzed in biopsies obtained during endoscopy.

Therapeutic interference using KCNN4 channels openers might be protective in IBD. Opening of KCNN4 channels leads to stabilization of the epithelial barrier. Thus, $\mathrm{K}^{+}$channels could be a promising novel target for IBD therapy due to the beneficial effects of KCNN4 openers on epithelial restitution.

Conflicts of interest: None to declare.

Authors' contribution: C.S. performed experiments, analyzed data and discussed the results. L.B. critically revised and edited the manuscript. K.P. performed additional experiments and analyzed data. C.K., K.G. and M.M. provided critical feedback and edited the manuscript. G.W. initiated, designed, guided the research, discussed results, wrote the paper and edited the manuscript. All authors critically revised the manuscript and approved the final version to be published.

Acknowledgements: The authors thank the patients with inflammatory bowel disease, diverticulitis or colorectal carcinoma for surgical specimen donations. We thank the Departments of Surgery (University of Regensburg, Hospital Barmherzige Brueder and Hospital St. Josef, Regensburg) and the Institute of Pathology (University of Regensburg) for providing surgical specimens. Further, we thank S. Zundler (University of Erlangen) for sharing samples.

Supplementary material: To access the supplementary material visit the online version of the J Gastrointestin Liver Dis at http://dx.doi. org/10.15403/jgld-903.

\section{REFERENCES}

1. van Limbergen J, Wilson DC, Satsangi J. The genetics of Crohn's disease. Annu Rev Genomics Hum Genet 2009;10:89-116. doi:10.1146/ annurev-genom-082908-150013

2. Kaser A, Zeissig S, Blumberg RS. Genes and environment: how will our concepts on the pathophysiology of IBD develop in the future? Dig Dis 2010;28:395-405. doi:10.1159/000320393

3. Round JL, Mazmanian SK. The gut microbiota shapes intestinal immune responses during health and disease. Nat Rev Immunol 2009;9:313-323. doi:10.1038/nri2515

4. Loh G, Blaut M. Role of commensal gut bacteria in inflammatory bowel diseases. Gut Microbes 2012;3:544-555. doi:10.4161/gmic.22156

5. Niessner M, Volk BA. Altered Th1/Th2 cytokine profiles in the intestinal mucosa of patients with inflammatory bowel disease as assessed by quantitative reversed transcribed polymerase chain reaction (RT-PCR). Clin Exp Immunol 1995;101:428-435. doi:10.1111/j.1365-2249.1995. tb03130.x

6. Obermeier F, Kojouharoff G, Hans W, Scholmerich J, Gross V, Falk W. Interferon-gamma (IFN-gamma)- and tumour necrosis factor (TNF)induced nitric oxide as toxic effector molecule in chronic dextran sulphate sodium (DSS)-induced colitis in mice. Clin Exp Immunol 1999;116:238-245. doi:10.1046/j.1365-2249.1999.00878.x

7. Sasaki T, Hiwatashi N, Yamazaki H, Noguchi M, Toyota T. The role of interferon gamma in the pathogenesis of Crohn's disease. Gastroenterol Jpn 1992;27:29-36.

8. Fuss IJ, Neurath M, Boirivant M, et al. Disparate CD4+ lamina propria (LP) lymphokine secretion profiles in inflammatory bowel disease. Crohn's disease LP cells manifest increased secretion of IFN-gamma, whereas ulcerative colitis LP cells manifest increased secretion of IL-5. J Immunol 1996;157:1261-1270.

9. Liang GH, Weber CR. Molecular aspects of tight junction barrier function. Curr Opin Pharmacol 2014;19:84-89. doi:10.1016/j. coph.2014.07.017

10. Bhat AA, Uppada S, Achkar IW, et al. Tight Junction Proteins and Signaling Pathways in Cancer and Inflammation: A Functional Crosstalk. Front Physiol 2018;9:1942. doi:10.3389/fphys.2018.01942

11. Boivin MA, Roy PK, Bradley A, Kennedy JC, Rihani T, Ma TY. Mechanism of interferon-gamma-induced increase in T84 intestinal epithelial tight junction. J Interferon Cytokine Res 2009;29:45-54 doi:10.1089/jir.2008.0128

12. Baumgart DC, Dignass AU. Intestinal barrier function. Curr Opin Clin Nutr Metab Care 2002;5:685-694. doi:10.1097/00075197-20021100000012

13. Wilson AJ, Gibson PR. Role of epidermal growth factor receptor in basal and stimulated colonic epithelial cell migration in vitro. Exp Cell Res 1999;250:187-196. doi:10.1006/excr.1999.4496

14. Paul G, Marchelletta RR, McCole DF, Barrett KE. Interferon-gamma alters downstream signaling originating from epidermal growth factor receptor in intestinal epithelial cells: functional consequences for ion transport. J Biol Chem 2012;287:2144-2155. doi:10.1074/jbc. M111.318139

15. De Mey JR, Freund JN. Understanding epithelial homeostasis in the intestine: An old battlefield of ideas, recent breakthroughs and remaining controversies. Tissue Barriers 2013;1:e24965. doi:10.4161/ tisb.24965

16. Caricilli AM, Castoldi A, Camara NOS. Intestinal barrier: A gentlemen's agreement between microbiota and immunity. World J Gastrointest Pathophysiol 2014;5:18-32. doi:10.4291/wjgp.v5.i1.18 
17. Nanda Kumar NS, Singh SK, Rajendran VM. Mucosal potassium efflux mediated via Kcnn4 channels provides the driving force for electrogenic anion secretion in colon. Am J Physiol Gastrointest Liver Physiol 2010;299:G707-G714. doi:10.1152/ajpgi.00101.2010

18. Warth R. Potassium channels in epithelial transport. Pflugers Arch 2003;446:505-513. doi:10.1007/s00424-003-1075-2

19. Tharp DL, Bowles DK. The intermediate-conductance Ca2+-activated $\mathrm{K}+$ channel (KCa3.1) in vascular disease. Cardiovasc Hematol Agents Med Chem 2009;7:1-11. doi:10.2174/187152509787047649

20. Scharl M, Paul G, Barrett KE, McCole DF. AMP-activated protein kinase mediates the interferon-gamma-induced decrease in intestinal epithelial barrier function. J Biol Chem 2009;284:27952-27963. doi:10.1074/jbc M109.046292

21. Zundler S, Caioni M, Muller M, Strauch U, Kunst C, Woelfel G. K+ Channel Inhibition Differentially Regulates Migration of Intestinal Epithelial Cells in Inflamed vs. Non-Inflamed Conditions in a PI3K/ Akt-Mediated Manner. PLoS One 2016;11:e0147736. doi:10.1371/ journal.pone.0147736

22. Podolsky DK. Inflammatory bowel disease. N Engl J Med 2002;347:417429. doi:10.1056/NEJMra020831

23. McCole DF. IBD candidate genes and intestinal barrier regulation. Inflamm Bowel Dis 2014;20:1829-1849. doi:10.1097/ MIB.0000000000000090

24. McCole DF. Regulation of epithelial barrier function by the inflammatory bowel disease candidate gene, PTPN2. Ann N Y Acad Sci 2012;1257:108-114. doi:10.1111/j.1749-6632.2012.06522.x

25. Rajasekaran SA, Beyenbach KW, Rajasekaran AK. Interactions of tight junctions with membrane channels and transporters. BiochimBiophys Acta 2008;1778:757-769. doi:10.1016/j.bbamem.2007.11.007

26. Rajasekaran SA, Barwe SP, Gopal J, Ryazantsev S, Schneeberger EE Rajasekaran AK. Na-K-ATPase regulates tight junction permeability through occludin phosphorylation in pancreatic epithelial cells. Am J PhysiolGastrointest Liver Physiol 2007;292:G124-G133. doi:10.1152/ ajpgi.00297.2006

27. Strobaek D, Brown DT, Jenkins DP, et al. NS6180, a new K(Ca) 3.1 channel inhibitor prevents T-cell activation and inflammation in a rat model of inflammatory bowel disease. Br J Pharmacol 2013;168:432444. doi:10.1111/j.1476-5381.2012.02143.x

28. Ohya S, Fukuyo Y, Kito H, et al. Upregulation of KCa3.1 K(+) channel in mesenteric lymph node CD4(+) T lymphocytes from a mouse model of dextran sodium sulfate-induced inflammatory bowel disease. Am J Physiol Gastrointest Liver Physiol 2014;306:G873-G885. doi:10.1152/ ajpgi.00156.2013

29. Preston P, Wartosch L, Gunzel D, et al. Disruption of the K+ channel beta-subunit KCNE3 reveals an important role in intestinal and tracheal Cl- transport. J Biol Chem 2010;285:7165-7175. doi:10.1074/jbc. M109.047829

30. Simms LA, Doecke JD, Roberts RL, et al. KCNN4 gene variant is associated with ileal Crohn's Disease in the Australian and New Zealand population. Am J Gastroenterol 2010;105:2209-2217. doi:10.1038/ajg.2010.161

31. Quaroni A, Wands J, Trelstad RL, Isselbacher KJ. Epithelioid cell cultures from rat small intestine. Characterization by morphologic and immunologic criteria. J Cell Biol 1979;80:248-265. doi:10.1083/ jcb.80.2.248

32. Basavappa S, Vulapalli SR, Zhang H, Yule D, Coon S, Sundaram U. Chloride channels in the small intestinal cell line IEC-18. J Cell Physiol 2005;202:21-31. doi:10.1002/jcp.20085

33. Basalingappa KM, Rajendran VM, Wonderlin WF. Characteristics of Kcnn 4 channels in the apical membranes of an intestinal epithelial cell line. Am J Physiol Gastrointest Liver Physiol 2011;301:G905-G911. doi:10.1152/ajpgi.00558.2010

34. Hofmann C, Obermeier F, Artinger M, et al. Cell-cell contacts prevent anoikis in primary human colonic epithelial cells. Gastroenterology 2007;132:587-600. doi:10.1053/j.gastro.2006.11.017

35. Grossmann J, Walther K, Artinger M, et al. Progress on isolation and short-term ex-vivo culture of highly purified non-apoptotic human intestinal epithelial cells (IEC). Eur J Cell Biol 2003;82:262-270. doi:10.1078/0171-9335-00312

36. Livak KJ, Schmittgen TD. Analysis of relative gene expression data using real-time quantitative PCR and the 2(-Delta Delta C(T)) Method. Methods 2001;25:402-408. doi:10.1006/meth.2001.1262

37 Pillozzi S, D'Amico M, Bartoli G, et al. The combined activation of K Ca 3.1 and inhibition of K v 11.1/hERG1 currents contribute to overcome Cisplatin resistance in colorectal cancer cells. Br J Cancer 2018;118:200-212. doi:10.1038/bjc.2017.392

38. Duan T, Cil O, Thiagarajah JR, Verkman AS. Intestinal epithelial potassium channels and CFTR chloride channels activated in ErbB tyrosine kinase inhibitor diarrhea. JCI Insight 2019;4:e126444. doi:10.1172/jci.insight.126444

39. Jantarajit W, Lertsuwan K, Teerapornpuntakit J, Krishnamra N, Charoenphandhu N. CFTR-mediated anion secretion across intestinal epithelium-like Caco-2 monolayer under PTH stimulation is dependent on intermediate conductance K+ channels. Am J Physiol Cell Physiol 2017;313:C118-C129. doi:10.1152/ajpcell.00010.2017

40. Agarwal JJ, Zhu Y, Zahang QY, Mongin AA, Hough LB. TRAM-34, a putatively selective blocker of intermediate-conductance, calciumactivated potassium channels, inhibits cytochrome $\mathrm{P} 450$ activity. PloS One 2013;8:e63028. doi:10.1371/journal.pone.0063028

41. Zhang W, Ramamoorthy Y, Kilicarslan T, Nolte H, Tyndale RF, Sellers EM. Inhibition of cytochromes $\mathrm{P} 450$ by antifungal imidazole derivatives. Drug Metab Dispos 2002;30:314-318. doi:10.1124/dmd.30.3.314

42. Roger S, Potier M, Vandier C, Le Guennec JY, Besson P. Description and role in proliferation of iberiotoxin-sensitive currents in different human mammary epithelial normal and cancerous cells. Biochim Biophys Acta 2004;1667:190-199. doi:10.1016/j.bbamem.2004.10.002

43. Galvez A, Gimenez-Gallego G, Reuben JP, et al. Purification and characterization of a unique, potent, peptidyl probe for the high conductance calcium-activated potassium channel from venom of the scorpion Buthustamulus. J Biol Chem 1990;265:11083-11090.

44. Madara JL. Loosening tight junctions. Lessons from the intestine. J Clin Invest 1989;83:1089-1094. doi:10.1172/JCI113987

45. Scharl M, Paul G, Weber A, et al. Protection of epithelial barrier function by the Crohn's disease associated gene protein tyrosine phosphatase N2. Gastroenterology 2009;137:2030-2040.e5. doi:10.1053/j. gastro.2009.07.078

46. Sandle GI. Pathogenesis of diarrhea in ulcerative colitis: new views on an old problem. J Clin Gastroenterol 2005;39:S49-S52. doi:10.1097/01. mcg.0000155520.04253.37

47. Malerba M, Radaeli A, Mancuso S, Pelosa R. The potential therapeutic role of potassium channel modulatirs in asthma and chronic obstructive pulmonary disease. J Biol Reg Homeost Agents 2010;24:123-130.

48. Hansen LK. The role of T cell potassium channels, KV1.3 and $\mathrm{KCa} 3.1$, in the inflammatory cascade in ulcerative colitis. Dan Med J 2014;61:B4946.

49 Koch Hansen L, Sevelsted-Moller L, Rabjerg M, et al. Expression of T-cell KV1.3 potassium channel correlates with pro-inflammatory cytokines and disease activity in ulcerative colitis. J Crohns Colitis 2014;8:1378-1391. doi:10.1016/j.crohns.2014.04.003 
50. Magalhaes D, Cabral JM, Soares-da-Silva P, Magro F. Role of epithelial ion transports in inflammatory bowel disease. Am J Physiol Gastrointest Liver Physiol 2016;310:G460-G476. doi:10.1152/ajpgi.00369.2015

51. Orban C, Szabo D, Bajnok A, et al. Altered activation of peripheral CD8+ T cells in pediatric Crohn's disease. Immunol Lett 2017;185:48-51. doi:10.1016/j.imlet.2017.03.009

52. Orban C, Szabo D, Bajnok A, et al. Altered calcium influx of peripheral Th2 cells in pediatric Crohn's disease: infliximab may normalize activation patterns. Oncotarget 2016;7:44966-44974. doi:10.18632/ oncotarget. 10036
53. Di L, Srivastava S, Zhdanova O, et al. Inhibition of the $\mathrm{K}+$ channel KCa3.1 ameliorates T cell-mediated colitis. Proc Natl Acad Sci U S A 2010;107:1541-1546. doi:10.1073/pnas.0910133107

54. Watson CJ, Hoare CJ, Garrod DR, Carlson GL, Warhurst G. Interferongamma selectively increases epithelial permeability to large molecules by activating different populations of paracellular pores. J Cell Sci 2005;118:5221-5230. doi:10.1242/jcs.02630

55. Huang H, Liu JQ, Yu Y, et al. Regulation of TWIK-related potassium channel-1 (Trek1) restitutes intestinal epithelial barrier function. Cell Mol Immunol 2016;13:110-118. doi:10.1038/cmi.2014.137 